\title{
Platelet-Rich plasma injections treatment in patients having early osteoarthritis of knee joint.
}

1. MBBS, FCPS (Medicine) Assistant Professor Medicine Quaid e Azam Medical College/ Bahawal Victoria Hospital, Tahira Jamshed Medical Complex Bahawalpur, Pakistan.

2. MBBS, FCPS (Medicine), MCPS-HPE

Professor \& Head Medicine Quaid e Azam Medical College/ Bahawal Victoria Hospital Civil Hospital, Bahawalpur, Pakistan

3. MBBS, FCPS (Medicine)

Assistant Professor Medicine Quaid e Azam Medical College/ Bahawal Victoria Hospital, Bahawalpur, Pakistan.

Correspondence Address: Dr. Ali Imran

Assistant Professor Medicine Quaid e Azam Medical College / Bahawal Victoria Hospital,

Tahira Jamshed Medical Complex Bahawalpur, Pakistan.

iimranali@yahoo.com

Article received on: 03/09/2020

Accepted for publication:

$09 / 12 / 2020$

\section{Ali Imran', Qazi Masroor Ali $^{2}$, Syed Hashim Raza ${ }^{3}$}

ABSTRACT... Objective: To find out the outcome following intra-articular platelet-rich plasma (PRP) injection in patients having early OA of knee. Study Design: Prospective Study. Setting: Tahira Jamshed Medical Complex, Bahawalpur. Period: January 2019 to February 2020. Material \& Methods: A total of 112 cases of both gender, aged 40 to 60 years, Grade I and Grade II OA, having symptoms of OA for a minimum duration of 1 year, were enrolled. All participants were assessed for physical activity and pain linked with arthritis as per "Western Ontario and McMaster University Arthritis Index (WOMAC) score". Results: Out of a total 98 cases who completed the study, majority 58 (59.2\%) were female, 67 (68.4\%) had KellgrenLawrence Grade-I OA and 81 (82.7\%) were experiencing OA symptoms with a duration of less than 2 years. The difference between mean WOMAC score before the treatment $(80.38+6.3)$ and after PRP treatment $(42.93+6.9)$ turned out to be statistically significant ( $p$ value $<0.001)$. After the treatment, cases having duration of symptoms of OA as less than 2 years had significantly less WOMAC score ( $p$ value $=0.0418$ ). Conclusion: PRP treatment in patients having early OA seemed to be quite efficacious. The effects of PRP injections were more favorable in cases having clinical symptoms of $\mathrm{OA}$ for less than 2 years of duration.

Key words: $\quad$ Knee, Osteoarthritis, Platelet-Rich Plasma Injection, Treatment.

Article Citation: Imran A, Ali QM, Raza SH. Platelet-Rich plasma injections treatment in patients having early osteoarthritis of knee joint. Professional Med J 2021; 28(8):1132-1136. https://doi.org/10.29309/TPMJ/2021.28.08.6060

\section{INTRODUCTION}

Osteoarthritis $(\mathrm{OA})$ is known to be the commonest articular disorder resulting from defects in articular cartilage. Not only OA is a major reason of musculoskeletal disability but also poses significant reduction to quality of life (QoL). ${ }^{1} \mathrm{OA}$ can influence any of the joints but most commonly it is observed in knees, hips, hand and feet. ${ }^{2}$ Increasing age increase the overall prevalence of osteoarthritis while age between 55-64 years has been found to be mostly affected. ${ }^{3}$

OA does not only cause wear and tear to cartilage but it is described as a complex disorder. Inflammatory mediators like inflammatory cytokines are thought to play a major role in underlying pathophysiology of OA. ${ }^{4}$ Joint damage resulting from $O A$ results due to disparity between breakdown and repair of joint tissues along with cellular alterations and bio-chemical stress cause various other secondary alterations in the affected joint. With ageing populations and increase in obesity around the world, number of knee joint surgeries/replacements are bound to increase significantly in the future. ${ }^{5}$ Knee replacement is considered to be a costly method for patients of OA so any alternative options for the treatment of OA of knee is thought to be worthy. ${ }^{6}$

For treating $\mathrm{OA}$, quite a few main bio-chemical pathways have been acknowledged by researchers around the world. Aiming relief from the symptoms of $O A$, supportive management like analgesics, non-steroidal anti-inflammatory drugs (NSAIDs), viscosupplementations and intra-articular corticosteroids are widely adopted. ${ }^{5}$ Intra-articular hyaluronic acid has also been in use for a long while more recently, platelet-rich plasma (PRP) have attracted the interest of many around the world for treating OA. ${ }^{7,8}$

PRP is derived from autologous blood that 
contains growth factors known to activate the healing process in damaged cartilage. ${ }^{9}$ PRP is also thought to help tissue regeneration. PRP has been considered as a more effective option treating $\mathrm{OA}$ in comparison to options like hyalouronic acid injections but scarcity of local data exist to analyze the outcomes in our patients. ${ }^{10}$ As no study has been conducted in South Punjab to observe the outcome of PRP injections before, this study was planned to find out the outcome following intra-articular PRP injection in patients having OA of knee.

\section{MATERIAL \& METHODS}

This prospective study was conducted from January 2019 to February 2020, at Tahira Jamshed Medical Complex, Bahawalpur was the venue of this study. Approval from Institutional Ethical Board was taken for this study. A total of 112 cases of both gender, aged 40 to 60 years, having Grade I and Grade II OA were enrolled. All cases had symptoms of OA for a minimum duration of 6 months, hemoglobin levels of more than $12 \mathrm{~g} / \mathrm{dL}$ and platelets more than $150000 \mathrm{~mL}$. All the enrolled cases had failure of pharmacological therapies as well as physiotherapy for considerable long term relief of their OA related symptoms. Patients having diabetes, infection or trauma, with autoimmune disorders or platelet abnormalities, or having any kind of malignancy were not included.

All enrolled patients were detailed about the procedure as well as possible benefits and adverse outcomes related with this study. Witten consent was taken from all the participants. All the study data like demographical information, occupation, body mass index, blood grouping, grade as well as duration of arthritis was recorded. All the participants were assessed for physical activity and pain linked with arthritis as per "Western Ontario and McMaster University Arthritis Index (WOMAC) score". ${ }^{11}$

Aiming preparation of PRP utilizing differential centrifugation, PRP method and buffy-coat method have usually been in use by other researchers. ${ }^{12}$ We opted for PRP method. To make 4 to $4 \mathrm{ml}$ of PRP, the process included drawing of blood from cubital vein of the patient. Collection of blood samples was made in bags having anti-coagulant (citrate phosphate dextrose and adenine). After that, collected samples were shifted to a tube for centrifugation at a constant acceleration aiming separation of red blood cells. As 3 layers (platelets with white blood cells, buffy coat rich in white blood cells, and red blood cells from top to bottoms) are attained, upper layer and superficial buffy coat are centrifuged again in a new sterile tube sufficient enough to form soft pellets at the bottom. Upper $2 / 3^{\text {rd }}$ volume is discarded whereas what remained $\left(1 / 3^{\text {rd }}\right)$ is homogenized to form PRP. PRP was dispensed in a sterile syringe. Cases were allotted appointments in OPD for the purpose of PRP injection. ${ }^{13}$ Under aseptic conditions, PRP got injected in the knee through supralateral approach in suprapatellar pouch. Following the infection, observations were done for 20 minutes. After that, patients were discharged asking them to observe and note any signs of infection, pain, redness, warmth or any difficulty in bearing weight. Paracetamol was advised if any patient experienced pain while all cases were prohibited to use no steroidal anti-inflammatory drugs (NSAIDs) and steroids. Patients were followed up fortnightly while a total of 3 injections were administered at four weeks interval to every patient. WOMAC score was noted be at the time of enrollment for every patient in this study. WOMAC score was then compared at 6 months intervals for each patient to note the effects of PRP injections.

SPSS version 21.0 was adopted aiming data analysis. Datawasexhibitedintermsoffrequencies. Comparison of WOMAC scores before and after the treatment (as described above) was done utilizing paired $t$ test. Independent sample $t$ test was adopted to compare WOMAC between subgroups. $P$ value equal or less than 0.05 was taken as significant.

\section{RESULTS}

Out of a total of 112 cases, 98 completed the study while other 14 lost follow up. So, 98 patients were included in the final analysis for this study. There were 58 (59.2\%) female and 40 males (40.8\%). Overall, mean age was noted to be 53.37 years 
with standard deviation of 5.36 years. Majority of the cases, 67 (68.4\%) were having KellgrenLawrence Grade-I OA while 31 (31.6\%) had Grade-II. Duration of OA symptoms as more than 2 years was recorded in $17(17.3 \%)$ cases while remaining $81(82.7 \%)$ were experiencing OA symptoms with a duration of less than 2 years.

Mean WOMAC score before the treatment was 80.38 with a standard deviation of 6.3 while after PRP treatment, it was 42.93 with standard deviation of 6.9. The difference was noted to be statistically significant after PRP treatment ( $p$ value $<0.001$ ).
After the treatment, when WOMAC scores were compared in between subgroups, cases having duration of symptoms of OA as less than 2 years had significantly less WOMAC score as compared to those who had duration of OA symptoms for more than 2 years ( $p$ value $=0.0418)$. WOMAC scores between male and female or in between cases of Grade-I and Grade-II OA did not find to have any significant difference ( $p$ value $>0.05$ ). No major adverse events were reported in any of the cases during the study period. Hyperemia was noted in 4 (4.1\%) cases, managed by limiting activity along with applying ice.

\begin{tabular}{|l|c|c|}
\hline & WOMAC Score (Mean+SD) & P-Value \\
\hline Before the start of Treatment & $80.38+6.3$ & $<0.001$ \\
\hline After 6 Months Follow up & $42.93+6.9$ & \\
\hline
\end{tabular}

Table-I. WOMAC score before the start of treatment and at 6 months follow-up.

\begin{tabular}{|c|c|c|c|}
\hline & & WOMAC Score (Mean+SD) & P-Value \\
\hline \multirow{2}{*}{ Gender } & Male & $42.6+4.9$ & \multirow{2}{*}{0.5857} \\
\hline & Female & $43.22+5.9$ & \\
\hline \multirow{2}{*}{ Kelgren-Lawrence Grade } & 1 & $42.18+6.1$ & \multirow{2}{*}{0.1976} \\
\hline & II & $43.84+5.4$ & \\
\hline \multirow{2}{*}{ Duration of OA Symptoms (years) } & $<2$ & $42.10+5.0$ & \multirow{2}{*}{0.0418} \\
\hline & $>2$ & $44.94+5.9$ & \\
\hline
\end{tabular}

Table-II. WOMAC score after 6 months follow-up.

\section{DISCUSSION}

PRP has been in use since 1950s for maxillofacial and conditions associated with dermatological disorders. In the last few years, lots of research has been conducted regarding wide variety of PRP role in other specialties like rheumatology and orthopedics as PRP is thought to be effective along with having minimum undesired effects. ${ }^{14,15}$ Researchers in In-vitro trials found PRP as chondrocytes stimulant while synthesis of proteoglycan as well collagen in terms of histological properties were also noted which was very alike to usual hyaline cartilage..$^{16-19}$

In the present study, significant improvement was seen in clinical symptoms of OA. It was also seen that cases with OA symptoms for duration of less than 2 years had significantly more improvement than those with more than 2 years. Shadabad $\mathrm{HN}$ and colleagues $^{20}$ in a meta-analysis found
PRP to have better efficacy in comparison to its comparator (hyalouronic acid) for relieving symptoms of OA of knee.

Trials have also showed that response of treatment options like PRP is more pronounced in younger cases with shorter history of duration of symptoms. ${ }^{13,21}$ We also noted very similar findings in our study as after the treatment, when WOMAC scores were compared in between subgroups, cases having duration of symptoms of $O A$ as less than 2 years had significantly less WOMAC score as compared to those who had duration of OA symptoms for more than 2 years $(p$ value $=$ 0.0418).

A randomized controlled trial ${ }^{22}$ adopting double blind prospective design analyzed PRP injections as a single, 2 injections 3 weeks apart and a single saline injection found out that PRP cases were 
noted to have significantly improved outcomes at the end of the study period.

Another systemic review ${ }^{23}$ looking evaluating the role of PRP in degenerative OA of hip or knee noted that most patients improved pain and functional outcomes at the end of 6 months while none of the patients reported worsening of scores.

A local study ${ }^{13}$ analyzing 214 patients with knee OA noted that mean WOMAC score before PRP treatment was noted to be 83.1 while after PRP treatment of 6 months, it came down to 38.8 ( $p$ value $<0.001)$. These results were very similar to what was found in the current research.

PRP treatment is minimally invasive along with being simple and beneficial. Data has come out advocating PRP to improve quality of life, improving symptoms of pain and enhancing knee functions. ${ }^{24} \mathrm{~A}$ study evaluating patients of knee OA recorded significant betterment at 6 and 12 months period with the use of PRP treatment. They also noted more improved results in cases who had mild to moderate OA. ${ }^{25}$ In the present study, we also had cases with early arthritis.

In the present study, no major adverse events were reported in any of the cases during the study period. Hyperemia was noted in 4 (4.1\%) cases which were managed by limiting activity along with applying ice. In the past, complications of mild severity like nausea or dizziness have been seen in patients treated with PRP. ${ }^{13,22}$

This is the $1^{\text {st }}$ study from South Punjab, Pakistan that evaluated the role of PRP treatment in patients with $\mathrm{OA}$ of knee but there were few limitations as well. We did not have any controls or comparison group in the current study which would further strengthen the results of our findings. Another limitation of this study was that we did not have a longer follow up which would enable us to see long term outcomes of PRP treatment.

\section{CONCLUSION}

PRP treatment in patients having early OA seemed to be quite efficacious. The effects of PRP injections were more favorable in cases having clinical symptoms of OA for less than 2 years of duration.

\section{Copyright $@ 09$ Dec, 2020.}

\section{REFERENCES}

1. Raeissadat SA, Rayegani SM, Babaee M, Ghorbani E. The effect of platelet-rich plasma on pain, function, and quality of life of patients with knee osteoarthritis. Pain Res Treat 2013; 2013:165967.

2. Litwic A, Edwards MH, Dennison EM, Cooper C. Epidemiology and burden of osteoarthritis. Br Med Bull 2013; 105:185-199.

3. Deshpande BR, Katz JN, Solomon DH, Yelin EH, Hunter DJ, Messier SP. Number of persons with symptomatic knee osteoarthritis in the US: impact of race and ethnicity, age, sex and obesity. Arthritis Care Res (Hoboken) 2016; 68(12):1743-1750.

4. Wright EA, Katz JN, Cisternas MG, Kessler CL, Wagenseller A, Losina E. Impact of knee osteoarthritis on health care resource utilization in a US populationbased national sample. Med Care. 2010; 48(9):785791.

5. Michael JW, Schlu"ter-Brust KU, Eysel P. The epidemiology, etiology, diagnosis, and treatment of osteoarthritis of the knee. Dtsch Arztebl Int 2010; 107(9): 152-162.

6. Kellett CF, Boscainos PJ, Gross AE. Surgical options for articular defects of the knee. Expert Rev Med Devices 2006; 3:585-593.

7. Marmotti A, Rossi R, Castoldi F, Roveda E, Michielon G, Peretti GM. PRP and articular cartilage: A clinical update. Biomed Res Int 2015; 2015:542502.

8. Figueroa D, Figueroa F, Calvo R, Vaisman A, Ahumada $X$, Arellano $S$. Platelet-rich plasma use in anterior cruciate ligament surgery: Systematic review of the literature. Arthroscopy 2015; 31:981-988.

9. Griffin JW, Hadeed MM, Werner BC, Diduch DR, Carson $\mathrm{EW}$, Miller MD. Platelet-rich plasma in meniscal repair: Does augmentation improve surgical outcomes? Clin Orthop Relat Res 2015; 473:1665-1672.

10. Pujol N, Salle De Chou E, Boisrenoult P, Beaufils P. Platelet-rich plasma for open meniscal repair in young patients: Any benefit? Knee Surg Sports Traumatol Arthrosc 2015; 23:51-58.

11. McConnell S, Kolopack P, Davis AM. The Western Ontario and McMaster Universities Osteoarthritis Index (WOMAC): A review of its utility and measurement properties. Arthritis Rheum 2001; 45(5):453-461. 
12. Dhurat $R$, Sukesh $M$. Principles and methods of preparation of platelet-rich plasma: $A$ review and author's perspective. J Cutan Aesthet Surg 2014; 7(4):189-197.

13. Rasheed N, Hafeez K, Zaidi IH, Askari R, Rasheed $\mathrm{N}$, Khani GM. Role of platelet-rich plasma in early osteoarthritis of knee joint: Experience from a tertiary care center in Pakistan. J Orthop Surg. 2019; $27(2): 1-4$.

14. Marx RE. Platelet-rich plasma (PRP): What is PRP and what is not PRP? Implant Dent 2001; 10:225-228.

15. Tayapongsak P, O'Brien DA, Monteiro CB, ArceoDiaz LY. Autologous fibrin adhesive in mandibular reconstruction with particulate cancellous bone and marrow. J Oral Maxillofac Surg 1994; 52:161-165.

16. Milano G, Sanna PE, Deriu L, Careddu G, Manunta $L$, Manunta $A$. The effect of platelet rich plasma combined with microfractures on the treatment of chondral defects: an experimental study in a sheep model. Osteoarthritis Cartilage 2010; 18:971-980.

17. Kon E, Buda R, Filardo G, Di Martino A, Timoncini A, Cenacchi A. Platelet-rich plasma: Intra-articular knee injections produced favorable results on degenerative cartilage lesions. Knee Surg Sports Traumatol Arthrosc 2010; 18:472-479.

18. Krüger JP, Hondke $S$, Endres $M$, Pruss A, Siclari A, Kaps C. Human platelet-rich plasma stimulates migration and chondrogenic differentiation of human subchondral progenitor cells. J Orthop Res 2012; 30:845-852.
19. Lee HR, Park KM, Joung YK, Park KD, Do SH. Plateletrich plasma loaded in situ-formed hydrogel enhances hyaline cartilage regeneration by CB1 upregulation. J Biomed Mater Res A 2012; 100:3099-3107.

20. Sadabad HN, Behzadifar M, Arasteh F, Behzadifar M, Dehghan HR. Efficacy of platelet-rich plasma versus hyaluronic acid for treatment of knee osteoarthritis: A systematic review and meta-analysis. Electron Physician 2016; 8(3):2115-2122.

21. Jang SJ, Kim JD, Cha SS. Platelet-rich plasma (PRP) injections as an effective treatment for early osteoarthritis. Eur J Orthop Surg Traumatol 2013; 23(5):573-580.

22. Patel S, Dhillon MS, Aggarwal S, Marwaha N, Jain A. Treatment with platelet-rich plasma is more effective than placebo for knee osteoarthritis: A prospective, double-blind, randomized trial. Am J Sports Med 2013; 41:356-364.

23. Dold AP, Zywiel MG, Taylor DW, Dwyer T, Theodoropoulos $\mathrm{J}$. Platelet-rich plasma in the management of articular cartilage pathology: A systematic review. Clin J Sport Med 2014; 24:31-43.

24. Filardo G, Kon E, Buda R, et al. Platelet-rich plasma intra-articular knee injections for the treatment of degenerative cartilage lesions and osteoarthritis. Knee Surg Sports Traumatol Arthrosc 2011; 19:528-535

25. Kon E, Buda R, Filardo G, et al. Platelet-rich plasma: Intraarticular knee injections produced favorable results on degenerative cartilage lesions. Knee Surg Sports Traumatol Arthrosc 2010; 18(4):472-479.

\begin{tabular}{|c|c|c|c|}
\hline \multicolumn{4}{|c|}{ AUTHORSHIP AND CONTRIBUTION DECLARATION } \\
\hline Sr. \# & Author(s) Full Name & Contribution to the paper & Author(s) Signature \\
\hline 1 & Ali Imran & $\begin{array}{l}\text { Methodology, Data Collection, } \\
\text { Data Analysis. }\end{array}$ & \\
\hline 2 & Qazi Masroor Ali & $\begin{array}{l}\text { Literature Reivew, Proof reading, } \\
\text { Supervision. }\end{array}$ & \\
\hline 3 & Syed Hashim Raza & $\begin{array}{l}\text { Drafting, Discussion, Data } \\
\text { interpretation. }\end{array}$ & \\
\hline
\end{tabular}

